\title{
Atenção à saúde dos profissionais do sexo: a ótica da equipe de enfermagem da estratégia saúde da família
}

A prostituição é vista pelo Ministério do Trabalho e Emprego (MTE) como um trabalho onde o sexo é artigo de mercadoria, sendo realizado em troca de dinheiro, sem junção afetiva entre os envolvidos. O objetivo é caracterizar a atenção à saúde prestada pela equipe de Enfermagem da Estratégia de Saúde da Família a profissional do sexo. Trata-se de uma pesquisa de campo descritiva, de abordagem qualitativa, que seguiu os preceitos éticos da Resolução $466 / 2012$ do CNS Foram pesquisados enfermeiros e técnicos de enfermagem que atuam na Estratégia Saúde da Família. Os dados foram coletados por meio de questionário estruturado e as respostas foram analisadas em percentuais e categorias. Embora seja uma das profissões mais antigas do mundo, a prostituição ainda é malvista pela sociedade. Percebeu-se que os profissionais da enfermagem possuem diferentes barreiras para agregar essa população aos serviços de saúde, comprometendo assim a atenção à saúde preconizada pelos princípios e diretrizes do Sistema Único de Saúde. Observou-se que a atenção à saúde oferecida pela equipe de enfermagem é permeada por dificuldades de cunho social como o preconceito, e relacionadas à própria formação do profissional. Cabe destacar que as políticas públicas precisam ser aprimoradas, assim como a atualização dos processos de trabalho das equipes da Estratégia Saúde da Família, para garantia do direito à saúde dessa população. Evidenciou-se a necessidade de ampliar a percepção da equipe de enfermagem sobre este público, principalmente através do processo de trabalho, de forma que garantam a integralidade do cuidado.

Palavras-chave: Prostituição; Saúde; Enfermagem.

\section{Health care for sex professionals: the view of the nursing team of the family health strategy}

Prostitution is seen by the Ministry of Labor and Employment (MTE) as a job where sex is an article of merchandise, being carried out in exchange for money without affective joining between those involved. The objective is to characterize the health care provided by the Nursing team of the Family Health Strategy to sex workers. This is a descriptive field research, with a qualitative approach, which followed the ethical precepts of Resolution $466 / 2012$ of the CNS. Nurses and nursing technicians who work in the Family Health Strategy were surveyed. Data were collected through a structured questionnaire and responses were analyzed in percentages and categories. Although prostitution is one of the oldest professions in the world, it is still frowned upon by society. It was noticed that nursing professionals have different barriers to add this population to health services, thus compromising the health care recommended by the principles and guidelines of the Unified Health System. It was observed that the health care offered by the nursing team it is permeated by difficulties of a social nature such as prejudice, and related to the professional's own training. It is worth mentioning that public policies need to be improved, as well as updating the work processes of the Family Health Strategy teams, in order to guarantee the right to health of this population. The need to expand the perception of the nursing team about this public was evidenced, mainly through the work process, in order to guarantee the integrality of care.

Keywords: Prostitution; Health; Nursing.

Topic: Enfermagem em Saúde Pública

Reviewed anonymously in the process of blind peer

Patrícia Sabino dos Santos (iD)

Faculdade Guaraí, Brasil

http://lattes.cnpq.br/0889632104470251

http://orcid.org/0000-0001-8653-7826

patriciasabino27@hotmail.com

\section{Priscilla Gonçalves Ferreira (id}

Faculdade Guaraí, Brasil

http://lattes.cnpq.br/2556500982253558

http://orcid.org/0000-0001-8261-2366

priscilla.gferreira@outlook.com

\section{Rogério Carvalho de Figueredo (ic)}

Faculdade Guaraí, Brasil

http://lattes.cnpq.br/2845056129867931

http://orcid.org/0000-0003-3349-4812

rigoh1@live.com
Received: 26/06/2021

Approved: 29/07/2021

Leidiany Souza Silva (iD)

Faculdade Guaraí, Brasil

http://lattes.cnpq.br/1908517700214366

http://orcid.org/0000-0001-9734-3699

leidianysouza@hotmail.com

Renata Cristina Correia da Silva Amorim (ic

Universidad Columbia del Paraguay, Paraguai

http://lattes.cnpq.br/9940283425759292

http://orcid.org/0000-0002-7568-4182

renatacsamorim@gmail.com
Referencing this:

SANTOS, P. S.; FERREIRA, P. G.; FIGUEREDO, R. C.; SILVA, L. S.; AMORIM, R. C. C. S.. Atenção à saúde dos profissionais do sexo: a ótica da equipe de enfermagem da estratégia saúde da família. Scire Salutis, v.11, n.3, p.90-99, 2021. DOI:

http://doi.org/10.6008/CBPC2236-9600.2021.003.0012 


\section{INTRODUÇÃO}

A prostituição, seja ela feminina ou masculina, é reconhecida como uma das atividades de trabalho mais remotas no contexto histórico, com evidências da antiguidade de que ocorrem na Grécia e em Roma. Destaca-se a prostituição feminina como a mais evidente, em que o referido ato, todavia, vem sendo modificado com o passar do tempo, à medida que as mudanças acontecem na própria sociedade. Percebese que, conforme acontecem as mudanças econômicas e sociais, e ocorre o aprofundamento das desigualdades entre grupos sociais, a prostituição ampliar-se, especialmente entre as mulheres, que acabam optando por se prostituir, esperando sobreviver com melhores condições de vida (PRADO et al., 2018).

Mesmo existindo desde os tempos mais remotos, a prostituição ainda hoje atravessa momentos de desequilíbrio quanto à valorização e o reconhecimento social, pois ao mesmo tempo em que esses profissionais são exaltados, em outros momentos são vistos como vítimas. De acordo com a Classificação Brasileira de Ocupações, os profissionais do sexo trabalham de forma autônoma, agindo em locais privados e/ou públicos, sendo capaz de atender e acompanhar clientes de ambos os sexos, com distintas orientações sexuais (CRUZ et al., 2016).

A prostituição é vista pelo Ministério do Trabalho e Emprego (MTE) como um trabalho onde o sexo é artigo de mercadoria, sendo realizado em troca de dinheiro, sem junção afetiva entre os envolvidos. Apontados pelo MTE como profissionais do sexo, essas pessoas seguem marginalizadas, discriminadas e enfrentam diferentes tipos de preconceitos. Em outra perspectiva, podemos ver que o conhecimento sobre a prostituição pode se transformar, o que depende diretamente das referências socioculturais e de distintos contextos e discursos morais que tracejam suas razões e consequências (PENHA et al., 2015).

Frequentemente, a atividade da prostituição acontece de forma excluída e julgada pela sociedade, definida pela exposição a circunstâncias de risco, como por exemplo, violência sexual, física e psicológica, usa de drogas e álcool, vulnerabilidades individual e social, exposição a diferentes patologias, entre elas as Infecções Sexualmente Transmissíveis (IST). Esse conjunto inibe o desenvolvimento de condições propícias ao exercício da cidadania, fazendo com que os/as profissionais do sexo, além de sofrerem com o preconceito e a discriminação, acostumam-se com a exclusão diante da sociedade, dificultando ainda mais o acesso às ações e serviços de promoção a saúde (BELÉM et al., 2018).

É indispensável atentar-se para as questões que envolvem os diferentes tipos de gêneros que acabam optando pela prostituição. Estudos mostram que a inclusão da mulher no mercado de trabalho é mais árdua quando confrontada à categoria masculina. A situação fica ainda pior na realidade de mulheres transgêneros. Segundo uma pesquisa da Associação Nacional de Travestis (ANTRA), 90\% das travestis e transexuais usam da prostituição para sobreviver no Brasil. Neste caso pode-se garantir que a prostituição é quase a única opção para sobreviver. Mesmo que elas tenham a capacitação necessária para o preenchimento de uma vaga no mercado de trabalho, o preconceito é manifesto no andamento da contratação (LAPA, 2013).

Com base no levantamento prévio sobre a temática, surge a seguinte questão norteadora desse 
estudo: "Como é a atuação da equipe de Enfermagem aos profissionais do sexo considerando a invisibilidade social dessas pessoas e barreiras para acesso aos serviços de saúde?".

O estudo se justifica com base na necessidade em estar buscando ações e estratégias que ajudem os profissionais da saúde que, muitas vezes, não sabem como prestar uma assistência adequada e específica a esse público. Com essa dificuldade de inserção dos profissionais do sexo na sociedade, a equipe de Enfermagem acaba não sabendo como agir, ficando difícil a identificação dos problemas que eles enfrentam. Espera-se com esta pesquisa, que os profissionais de Enfermagem compreendam a importância de perceber esse grupo de pessoas em sua integralidade, muitas vezes ignorado pela sociedade e pelos serviços de saúde. E assim, sejam estimuladas a encontrar métodos mais eficazes para ir ao encontro dessas pessoas, fazendo com que se sintam confortáveis e seguras em receber seus cuidados. Fomentando o planejamento de ações estratégicas que possam garantir a prevenção de doenças, a promoção da saúde, além de apoio e reconhecimento como cidadãos. Esse artigo teve como objetivo caracterizar a atenção à saúde prestada pela equipe de Enfermagem da Estratégia de Saúde da Família às pessoas que trabalham como profissionais do sexo.

\section{METODOLOGIA}

Trata-se de uma pesquisa de campo descritiva, de abordagem qualitativa. A presente pesquisa seguiu os preceitos éticos da Resolução 466/2012 e foi aprovada pelo comitê de ética em pesquisa da Universidade Federal de Goiás (UFG), com o parecer número 3.653.441, CAAE: 22283119.2.0000.5083. A população deste estudo foi constituída por profissionais que compõem a equipe de enfermagem da Estratégia Saúde da Família (ESF) do município de Brasilândia do Tocantins-TO, sendo eles enfermeiros e técnicos em enfermagem. A amostra foi formada por 4 (quatro) enfermeiros e 8 (oito) técnicos em enfermagem, e se deu por conveniência.

A coleta de dados ocorreu em novembro de 2019, a partir da aplicação de um questionário sem identificação dos pesquisados, com 8 (oito) perguntas objetivas e 2 (dois) dissertativas. Devido à impossibilidade de adaptação de um questionário já validado e disponível na literatura, o questionário desse estudo foi elaborado pelos próprios autores, considerando os objetivos definidos, sendo aplicado antes da coleta de dados às profissionais da saúde, com perfil equivalente à população desse estudo, correspondendo a $50 \%$ do quantitativo da amostra. Essa aplicação prévia foi realizada para verificação da compreensão dos pesquisados e viabilidade de utilização.

Adotou-se como critérios de inclusão: profissionais de ambos os gêneros (masculino e feminino), que atuassem nas Unidades Básicas de Saúde (UBS) nas atividades de atenção direta aos usuários, que trabalhassem há pelo menos um mês na Estratégia Saúde da Família, que concordassem voluntariamente em participar desse estudo e assinassem de forma livre e consciente o Termo de Consentimento Livre e Esclarecido. Não puderam participar do estudo: demais profissionais que não faziam parte da equipe de enfermagem, que atuassem na UBS há menos de um mês, aqueles que não tinham contato direto com os usuários, e todos que não demonstraram interesse ou não aceitaram participar a partir do convite feito 
pelos pesquisadores.

Os dados coletados foram analisados de acordo com os formatos de perguntas do questionário, sendo as questões objetivas, representadas através de cálculos de porcentagem simples representados em gráficos e tabelas, e fundamentados teoricamente com auxílio de referenciais bibliográficos atualizados, com o objetivo de entender os resultados encontrados. Para as questões dissertativas, foi utilizado como referência o método proposto por Minayo ${ }^{5}$. As respostas dadas pelos participantes, foram identificadas de forma aleatória pelas iniciais ENF1, TEC1, ENF2, TEC2 sucessivamente, e foram organizadas de acordo com suas categorias por núcleo temático.

\section{RESULTADOS E DISCUSSÃO}

Enfermeiros e técnicos de enfermagem foram questionados sobre a frequência de atendimentos às profissionais do sexo no decorrer de suas jornadas de trabalho da ESF, e a sua percepção sobre o atendimento a esse público. Observa-se que 50\% dos profissionais pesquisados atendem de forma esporádica profissionais do sexo; 33\% afirmam que atendem frequentemente, e 17\% afirmam nunca terem atendido. Os dados apontam que o atendimento a esse público não é de rotina. Porém, é importante ressaltar, que as pessoas que se encontram nessa situação, nem sempre se identificam no momento do atendimento como profissionais do sexo. E devido à discriminação, preferem omitir essa informação de grande valor para a compreensão do estado de saúde e planejamento de atenção adequada.

Quando os participantes dessa pesquisa foram questionados sobre o gênero dos profissionais do sexo que eles atendiam, foi evidenciado que a maioria corresponde a mulheres (50\%), seguida de homens $(25 \%)$, e por pessoas trans (17\%). Ainda nesse contexto, $8 \%$ não responderam à questão. As principais dificuldades e desafios enfrentados pela equipe de enfermagem desse estudo foram identificadas nas questões dissertativas, a partir de questionamentos amplos, sendo eles "você sente dificuldade em atender profissionais do sexo? Quais são as dificuldades? E por quê?", apresentadas a seguir.

"Eu ainda tenho um pouco de receio em atender essas pessoas, não que eu não saiba, mas talvez eu não sei a forma mais correta de falar com elas." ENF1; "Eu não tenho preconceito, só acho que elas fazem isso por que querem, por que gosta disso." TEC1; "Em nenhuma disciplina de quando eu fiz faculdade, eu recebi alguma orientação de como atender esse tipo de pessoa. Tive que aprender quando comecei a trabalhar." ENF2; "Como a cidade é pequena, todos sabem quem faz isso por aqui. Por isso acho que elas ficam com vergonha de vim no postinho." TEC2; "Acho que minha maior dificuldade é atender sem transparecer que estou com preconceito ou julgando. Acho que elas devem pensar que estamos julgando o tempo todo." ENF3; "Tenho dificuldade em falar sobre prostituição." TEC3; "Às vezes nem todo mundo da equipe sabe guardar segredo sobre a paciente que faz isso, por isso ela não procura o posto de saúde." TEC4

As respostas foram tabuladas e organizadas de acordo com suas categorias temáticas e termos de evidência, conforme tabela 1 a seguir.

A partir da categorização das respostas, é possível perceber que as principais dificuldades e desafios para a equipe de enfermagem frente aos profissionais do sexo estão relacionadas à insegurança na abordagem; preconceito e desinformação sobre o tema; fragilidades no processo de trabalho da equipe e ausência do tema no decorrer de suas formações profissionais. Sabendo da importância da formação 
profissional e do seu reflexo na assistência prestada, questionou-se à equipe de enfermagem participante da pesquisa se eles já tinham participado de algum curso ou capacitação acerca da temática prostituição, e 83\% deles afirmaram que não. Esse dado reforça a importância da continuidade da formação profissional e da constante atualização, visto as diversas mudanças tanto na sociedade como nas políticas de saúde, exigindo assim novas condutas e abordagens de atenção à saúde.

Tabela 1: Categorias temáticas das respostas dissertativas sobre as principais dificuldades e desafios no atendimento às profissionais do sexo

\begin{tabular}{llc}
\multicolumn{1}{c}{ Categorias temáticas } & \multicolumn{1}{c}{$\begin{array}{c}\text { Termos de evidência } \\
\text { Identificação das }\end{array}$} \\
\hline $\begin{array}{ll}\text { Formação profissional que não } \\
\text { contempla esse público }\end{array}$ & $\begin{array}{l}\text { Nenhuma disciplina da faculdade recebi orientação sobre esse } \\
\text { público. }\end{array}$ & $\begin{array}{l}\text { ENF2 } \\
\text { guade pequena, todos sabem quem faz isso aqui; Equipe não sabe }\end{array}$ \\
\hline Processo de trabalho fragilizado & TEC; TEC4 \\
$\begin{array}{ll}\text { Preconceito e desinformação sobre } \\
\text { o tema }\end{array}$ & $\begin{array}{l}\text { Receio em atender essas pessoas; Eu não tenho preconceito. } \\
\text { Insegurança na abordagem }\end{array}$ & $\begin{array}{l}\text { Forma mais correta de falar; Atender sem transparecer que estou } \\
\text { com preconceito; dificuldade em falar sobre prostituição. }\end{array}$ \\
\hline
\end{tabular}

Quando questionado se a equipe de enfermagem possuía algum planejamento ou estratégia para atender os profissionais do sexo, todos afirmaram que até o momento não tinham nenhuma atividade planejada nem executada anteriormente que fosse direcionada para tal público. Justificaram afirmando que todas as atividades de educação em saúde sobre planejamento familiar e infecções sexualmente transmissíveis já contemplavam esse público, além do tratamento de algumas doenças adquiridas pelo sexo desprotegido conforme respostas a seguir:

\begin{abstract}
"Nós planejamos ações de acordo com a nossa agenda da semana. Temos dia de atendimento pra todos os públicos, mas nenhum específico para profissionais do sexo." ENF1; "Nunca fizemos uma palestra sobre profissional do sexo. Mas sempre damos camisinha no posto de saúde." TEC1; "Quando fazemos palestras sobre DST, gravidez na adolescência e planejamento familiar atendemos essas pessoas de forma indireta." ENF2; "Não acredito que dá certo fazer ações diretamente para profissional do sexo, porque elas não vão vir no posto de saúde por vergonha." TEC2; "Não acho necessário fazer ações específicas, pois já temos muitas ações para cada público." ENF3; "Já fizemos busca ativa de uma paciente que pegou DST, pois ela precisava terminar o tratamento." TEC2; "Depois dessa pesquisa acho que vamos incluir o tema em algumas de nossas ações, pois o nosso trabalho é para todos." ENF4
\end{abstract}

As respostas dos pesquisados evidenciam uma preocupante invisibilidade dos profissionais do sexo por parte da equipe de enfermagem, visto que eles só são contemplados de forma indireta, e acerca de temáticas induzidas por julgamento social, principalmente das infecções sexualmente transmissíveis. Vale ressaltar, que esse público não se restringe apenas à prática sexual e seus desfechos, mas também todo um contexto de exposição de danos à saúde e barreiras de apoio e na assistência.

No que tange a vivência da equipe de enfermagem no cuidar de profissionais do sexo e considerando os dados apresentados anteriormente, percebe-se que alguns estudos abordam sobre a qualidade da assistência prestada que, ainda existe a falta de um atendimento regular dos profissionais da Estratégia Saúde da Família. Além da abordagem por meio de atendimentos e procedimentos curativistas, sendo o exame de prevenção de colo de útero o mais frequente (PENHA et al., 2015).

Geralmente, a prostituição ocorre de forma marginalizada e estigmatizada pela sociedade, sendo as 
pessoas que se encontram nessa situação expostas a diferentes riscos à saúde, além das vulnerabilidades sociais, e devido a esse contexto, torna-se difícil o acesso aos serviços de saúde, ineficiente o processo de cuidar continuado, além de fragilizar as condições que propiciam o exercício da cidadania, e dessa forma, ficando invisíveis perante a sociedade e aos profissionais de saúde (BENOIT et al., 2017).

Nota-se que apesar dos diferentes gêneros, o maior percentual ainda é mulheres, devido à situação econômica precária, com insuficientes oportunidades de emprego, falta de formação e falta de conhecimento profissional. Por viverem em condições pouco valorizadas, ganhando bem menos que os homens, algumas mulheres foram levadas a buscar outros caminhos, entre eles, a prostituição (OLIVEIRA et al., 2017).

De acordo com um estudo feito por uma fundação francesa, que está sempre em busca de combater a exploração sexual, constata-se que chegam a 42 milhões o número de pessoas que se prostituem no mundo. Dessas pessoas, 75\% correspondem a mulheres jovens de 13 a 25 anos, sendo $90 \%$ negociadas através de cafetões ou cafetinas. Com base nessa realidade, muito se questiona sobre o que leva essas pessoas a escolherem este caminho, se por influência, necessidade, ou outros motivos. Também se considera como fator determinante, no caso de mulheres profissionais do sexo, o nascimento de um filho, onde a necessidade de sustento é relacionada com o início da atividade. Em resumo, prostituir-se representa para essas mulheres a melhor escolha para sobrevivência em ocasiões em que elas precisam manter a responsabilidade privativa de cuidarem e zelarem, sozinhas, de seus filhos (VILELA et al., 2015).

Estudos mostram que a procura pelos serviços de saúde no caso de mulheres profissionais do sexo, são mais frequentes do que os homens. E buscam a UBS devido o acontecimento de uma gravidez não planejada, para a realização do exame preventivo do colo uterino, na procura por preservativos e demais exames de rotina, por entenderam que é importante cuidar do próprio corpo, já que ele é instrumento de trabalho (ARAÚJO et al., 2014). Uma pesquisa realizada com profissionais do sexo destaca que a população de maior risco em contrair uma IST são mulheres mais jovens, pois é mais suscetível a fazer uso de substâncias psicoativas durante o ato sexual, aumentando assim a vulnerabilidade e o risco de contato com parceiro sem proteção, pois acabam não exigindo o uso de preservativo nas relações (VILELA et al., 2015).

Acerca das principais dificuldades e desafios no atendimento aos profissionais do sexo, a equipe de enfermagem precisa investigar a pessoa que se encontra nessa situação de forma integral, abrangendo seus aspectos bio-psico-socio-econômico-cultural, além das suas necessidades relativas ao estado de saúde. É necessário que estes profissionais sejam dotados de sensibilidade em suas condutas e atitudes, passando a enxergar o contexto de trabalho dentro da prostituição, com ênfase nos fatores que determinam agravos de saúde. Esse novo olhar, deve superar a visão biomédica e curativista no processo de cuidar, integrando a promoção da saúde, na perspectiva da integralidade (BELÉM et al., 2018).

Os profissionais de saúde ainda têm muita dificuldade em abordar alguns temas que envolve o assunto sexualidade, principalmente quando o público-alvo são profissionais do sexo. É necessário estarem preparados para lidarem com o preconceito, pensamentos rudes, mitos e tabus sobre sexualidade, saúde reprodutiva e acompanhamento dos filhos. Talvez os serviços pareçam ignorar que essa população existe, 
precisa e tem direito da assistência tanto quanto qualquer outro cidadão (DIAS et al., 2018).

De acordo com alguns estudos os indivíduos que se prostituem precisam lidar com alguns obstáculos para buscarem apoio ou ajuda quando necessitam, entre eles preconceito, tabu, e o medo da rejeição. Essas pessoas lidam com esses sentimentos constantemente e por medo de serem vítimas do que não é aceito e da coibição originária do seio da sociedade, muitos preferem esconder sua atividade profissional dos amigos, familiares e até mesmo dos seus parceiros, caso tenham. Contudo, existem também aqueles que admitem o seu papel na sociedade, estando em total concordância sobre os problemas que acabará enfrentando com a declaração de ser um/uma garoto/garota de programa (ARAÚJO et al., 2014; AQUINO et al., 2014; GARCIA et al., 2015).

Mesmo sendo uma das profissões mais antigas do mundo, a prostituição manifestada em meio à sociedade ainda recebe olhares e conclusões variadas, seja de preconceito ou reconhecimento, de afirmação ou negação. Levando em consideração o fato de que vivemos em uma sociedade imersa nas doutrinas e na moral do cristianismo, onde ainda se espera que a mulher deva se manter preservada convencionalmente como matriarca de uma família, e a mulher que usa seu corpo como instrumento de trabalho contraria as normas e os padrões pré-estabelecidos pela sociedade. E ao ir contra estes padrões, as pessoas que desempenham este tipo de atividade, onde o sexo é um produto de troca, sofrem com a estigmatização frequentemente (GARCIA et al., 2015). Na maioria das vezes o profissional do sexo não exerce sua função de cidadão por se sentirem menosprezadas, excluídas e discriminadas socialmente. Pesquisadores em saúde no Brasil afirmam que a própria saúde pública não fornece atenção devida quando se trata de profissional do sexo (SMAINIOTTO et al., 2018).

Para o profissional do sexo não comparecer a um serviço de saúde muitas vezes se dá pela dificuldade de acesso ou até mesmo falta de conhecimento de como funciona uma assistência voltada a eles, e acaba prejudicando a própria saúde. Então, cabe ao sistema de saúde assegurar a igualdade e equidade na qual todo cidadão brasileiro esteja incluído como seu favorecido (SMAINIOTTO et al., 2018).

O entendimento de que os serviços de saúde são procurados apenas quando ocorre à incidência de IST, diminui as expectativas de planos de cuidados pelos profissionais de saúde (VILELA et al., 2015). Esse tipo de situação acaba limitando a comunicação e a percepção no dia a dia, principalmente no cuidado integral à saúde, visto que essa população é alvo de fragilidade individual, social e programática em saúde além da dimensão sexual (BELÉM et al., 2018).

De acordo com todos os aspectos e devido à complexidade que estão ligados a prática da prostituição, torna-se imprescindível entender as carências das pessoas inseridas nesse exercício para só depois enumerar ações e estratégias que compreendam direitos sociais, sexuais e trabalhistas, valorização, conscientização em saúde e desenvolvimento de vínculo entre profissionais de saúde e profissionais do sexo (OVERS et al., 2013). E mesmo com toda a política do SUS ainda há necessidade de aperfeiçoamento para ações de prevenção voltadas a esse público, programar políticas de promoção da igualdade e estratégias que visem à diminuição de agravos que tornem possível a legitimação do direito à saúde e redução da maldade, desigualdade e disparidade que são associados a esses profissionais do sexo 
(MACHADO, 2017).

Sobre as estratégias de atenção à saúde dos profissionais do sexo e importância da equipe de enfermagem percebe-se uma formatação do modelo de atenção à saúde, popularmente conhecido como "modelo engessado", no qual se segue um programa e não um planejamento composto por estratégias e construído coletivamente a partir das necessidades da comunidade assistida. Além das ações pontuais, como busca ativa, que podem refletir ainda a abordagem biomédica e curativista, com pouca ênfase na promoção da saúde e prevenção de agravos.

Destaca-se ainda a necessidade de adaptar os horários e própria estrutura onde os serviços de saúde são realizados, buscando sempre capacitar e ampliar autonomia para que os profissionais de saúde prestarem assistência de maneira integral e contínua, tendo como princípio o respeito à liberdade de escolha e a não discriminação (COUTINHO et al., 2014) e, fundamentalmente, regularizar ponto específico que seja utilizado como porta de entrada da rede de atenção à saúde na qualidade de ambiente apropriado para obtenção de respostas resolutivas, atendendo qualquer necessidade básica de saúde desses profissionais do sexo por meio de inclusão em ações intersetoriais (ARAÚJO et al., 2014).

Conforme o exposto, ficou evidente que a saúde coletiva, na qualidade de instrumento de saberes e práticas, tem como objetivo programar políticas de promoção da igualdade e estratégias que visem à diminuição de agravos que tornem possível a legitimação do direito à saúde e redução da maldade, desigualdade e disparidade que são associados a esses profissionais do sexo. Estudos atestam que as estratégias para o atendimento a tal público ainda são escassas. E as políticas públicas mundiais priorizam ações que previnam o HIV/AIDS devido a ser considerada a causa do início da epidemia ocorrida no Brasil em 1996 e deixando ações de promoção e prevenção a outras doenças (AFONSO, 2014).

A fragmentação vinda das políticas públicas mundiais não se embasou em suscetibilidade às doenças e sim à dificuldade de acesso aos serviços de saúde, seja por horário de funcionamento ou por conscientização, desconsiderando questões inerentes a esses grupos no decorrer dos anos. Todavia, com tamanha estratificação da população feminina, tem um alto risco de as ações de saúde da mulher ficarem dispersas e perderem o foco principal. Com isso, as ações abrangem apenas o processo de reprodução humana, de atenção materno-infantil, e não a sexualidade e sua influência no estado de saúde (DIAS et al. 2018).

Dentre as estratégias de saúde desenvolvidas para profissionais do sexo destacam-se a disponibilização de métodos contraceptivos, camisinhas feminina e masculina, anticoncepcional. Os métodos são disponibilizados pela rede pública e são usados de forma livre e esclarecida. Faz-se necessário encaixar esses profissionais nos programas de saúde disponibilizados, também na rede pública como, por exemplo, o planejamento familiar, dando melhor condições de vida, fazendo rastreio e realizando orientações quanto tomadas de escolha com relação ao melhor método a ser utilizado. Tendo por base um acolhimento com escuta humanizada e igualitária (CRUZ et al., 2016).

A equipe de enfermagem possui ampla capacidade para facilitar a inserção dos profissionais do sexo no processo de cuidar oferecido nos serviços de saúde e principalmente na ESF, por esta representar a 
porta de entrada do SUS. Com a oferta de cuidados integrais, fortalecidos pelo vínculo entre profissional e paciente, essa população passará a ter o sentimento de pertencimento à rede de saúde, e que ela vá atender as suas reais necessidades, de forma eficiente e efetiva, e livre de preconceitos (CRUZ et al., 2016).

Um dos principais desafios para a ESF no seu campo de prática e saberes, ainda é a implementação de políticas de saúde para promoção da equidade, redução de danos, desigualdades e disparidades, e assim legitimar o direito à saúde das pessoas que se encontram nessa situação. Para isso, a equipe de enfermagem, classe majoritária entre os profissionais de saúde, desempenha função primordial diante desses desafios. Porém, se faz necessário diversas mudanças tanto a nível individual de cada profissional, como a nível coletivo, abrangendo o processo de trabalho e estrutura das Unidades Básicas de Saúde. Readequar a estrutura e os horários de atendimento, fomentar o desenvolvimento de competências e habilidades da equipe de enfermagem para alcance de cuidados integrais, contínuos, que respeitem a liberdade de escolha, e sem qualquer tipo de descriminação (BELÉM et al., 2018).

Vale ressaltar que existem políticas que norteiam a atuação dos profissionais de enfermagem para esse público e que visam à garantia do direito à saúde e a efetividade da atenção oferecida, entre elas: a Política de Atenção Integral à Saúde da Mulher, Política de Atenção Integral à Saúde do Homem, Política Nacional de Saúde Integral de Lésbicas, Gays, Bissexuais, Travestis e Transsexuais. Essas e outras políticas contribuem para superação dos obstáculos impostos para o cuidado com a saúde influenciados por desigualdades de gênero. Nesse sentido, o processo de construção e consolidação de políticas de saúde é completo e dinâmico, sendo materializado somente através de ações concretas em diversos setores, entre eles o da saúde (FERRAZ et al., 2010).

\section{CONCLUSÕES}

Percebem-se consideráveis mudanças sociais e políticas acerca da prostituição, porém, que não favoreceram o âmbito dos serviços de saúde. Observou-se que, o cuidado oferecido pela equipe de enfermagem às profissionais do sexo é permeado de diferentes dificuldades, desde as de cunho social como o preconceito, até as relacionadas à própria formação do profissional. Quanto aos desafios, percebese que os profissionais da enfermagem, devido seu contato direto com os seus clientes, precisam de estratégias e condutas que promovam a criação de vínculos entre profissionais e usuários, fortalecendo assim a segurança entre ambos. Aponta-se a necessidade de planejamento e organização do processo de trabalho da equipe que facilitem o acesso dessa população, e que promovam o cuidado integral e contínuo.

\section{REFERÊNCIAS}

AFONSO, M. L.. Regulamentar para quê(m)? As representações sociais de prostitutas sobre a regulamentação da profissão. Dissertação (Mestrado em Psicologia) - Universidade Federal de São Carlos, São Carlos, 2014.

AQUINO, O. S.; XIMENES, I. B.; PINHEIRO, A. K. B.. Políticas públicas de saúde voltadas à atenção à prostituta: breve resgate histórico. Enf. em foco, v.1, n.1, p.18-22, 2014 DOI: http://doi.org/10.21675/2357-707X.2010.v1.n1.4
ARAÚJO, O. D.; NERY, I. S.; MONTEIRO, C. F. S.; MOURA, M. E. B.. Representações sociais de mulheres profissionais do sexo. Cienc. Cuid. Saúde, v.13, n.4, p.714-721, 2014. DOI https://doi.org/10.4025/cienccuidsaude.v13i4.20380

BELÉM, J. M.; ALVES, M. J. H.; PEREIRA, E. V.; MAIS, E. R.; QUIRINO, G. S.; ALBUQUERQUE, G. A.. Prostituição e saúde: representações sociais de enfermeiros/as da estratégia saúde da família. Rev baiana enf, v.32, 2018. DOI: 


\section{http://dx.doi.org/10.18471/rbe.v32.25086}

BENOIT, C. S.; JANSSON, M.; SMITH, M.; FLAGG, J.. Prostitution stigma and its effect on the working conditions, personal lives, and health of sex workers. J Sex Res, v. 25 n.17, p.1-15, 2017. DOI: http://dx.doi.org/10.1080/00224499.2017.1393652

COUTINHO, J.; OLIVEIRA, A. L.. Redução de riscos no trabalho sexual em Portugal: representações dos técnicos interventores. Psic S. \& D, v.15, n.2, p.538-553, 2014. DOI: http://dx.doi.org/10.15309/14psd150216

CRUZ, N. L.; FERREIRA, C. L.; MARTINS, E.; SOUZA, M. O.. Cuidado com a saúde das mulheres profissionais do sexo: uma revisão narrativa. Disciplinarum Scientia, v.17, n.3, p. 339-352, 2016.

DIAS, I. H. P.; SILVA, M. R.; LEITE, E. P. R. C.; FREITAS, O. S.; SILVA, A. S.; CALHEIROS, C. A. P.. Assistência de enfermagem na Estratégia Saúde da Família quanto à sexualidade feminina. Cienc Cuid Saude, v.17, n.1, DOI: https://doi.org/10.4025/cienccuidsaude.v17i1.37811

FERRAZ, D.; KRAICZYK, J.. Gênero e Políticas Públicas de Saúde: construindo respostas para o enfrentamento das desigualdades no âmbito do SUS. Revista de Psicologia da UNESP, v.9, n.1, p.70-82, 2010.

GARCIA, O. R. Z.; LISBOA, L. C. S.. Consulta de enfermagem em sexualidade: um instrumento para assistência de enfermagem à saúde da mulher, em nível de atenção primária. Texto contexto enf., v.21, n.3, p.708-716, 2015. DOI: http://dx.doi.org/10.1590/S0104-07072012000300028

LAPA, N.. Mercado de trabalho para pessoas trans: realidade ou utopia no Brasil?. São Paulo, 2013.
MACHADO, J. P.. Proposta de regulamentação da prostituição no brasil: desmarginalização de uma profissão ou institucionalização da cafetinagem?. Monografia (Bacharelado em Direito) - Universidade Federal de Santa Catarina, Florianópolis, 2017.

OLIVEIRA, T. Z.; GUIMARÃES, L. V.; FERREIRA, D. P.. Mulher, prostituta e prostituição: da História ao Jardim do Éden, 2017.

OVERS, C.; LOFF, B.. Toward a legal frameworkthatpromotes and protects sex workeds' health and human rights. 2013.

PENHA, J. C.; AQUINO, C. B. Q.; NERO, E. A. R.; REIS, T. G. O. AQUINO, O. S.; PINHEIRO, A. K. B.. Fatores de risco para doenças sexualmente transmissíveis em profissionais do sexo do interior piauiense. Rev. Gaúcha Enferm, v.36, n.2, p.63-69, 2015. DOI: http://dx.doi.org/10.1590/1983$\underline{1447.2015 .02 .52089}$

PRADO, J. V. I.; AMARAL, F. B.; BARBOSA, Y. M.. Território da prostituição masculina em Goiânia: uma breve análise. Geografia em questão, v.11, n.1, p.75-88, 2018.

SMANIOTTO GEHLEN, R. G.; COSTA, C. M.; ARBOIT, J.; SILVA, E. B.. Situações de vulnerabilidade a violência vivenciadas por mulheres profissionais do sexo: estudo de caso. Cienc. Enferm, v.24, n.8, 2018

DOI:http://dx.doi.org/10.4067/s0717-95532018000100208

VILELA, W. V.; MONTEIRO, S.. Gênero, estigma e saúde: reflexões a partir da prostituição, do aborto e do hiv/aids entre mulheres. Epidemiol. Serv. Saúde, v.24, n.3, p.531540, 2015. DOI: https://doi.org/10.5123/S167949742015000300019

A CBPC - Companhia Brasileira de Produção Científica (CNPJ: 11.221.422/0001-03) detém os direitos materiais desta publicação. Os direitos referem-se à publicação do trabalho em qualquer parte do mundo, incluindo os direitos às renovações, expansões e disseminações da contribuiç̃o, bem como outros direitos subsidiários. Todos os trabalhos publicados eletronicamente poderão posteriormente ser publicados em coletâneas impressas sob coordenação da Sustenere Publishing, da Companhia Brasileira de Produção Científica e seus parceiros autorizados. Os (as) autores (as) preservam os direitos autorais, mas não têm permissão para a publicação da contribuição em outro meio, impresso ou digital, em português ou em tradução. 